Поздние недоношенные дети: эпидемиологические аспекты, заболеваемость, тактика ведения

\author{
А.Ф. Киосов \\ ГБУз «Областная клиническая больница № 2»; Россия, г. Челябинск
}

\begin{abstract}
Цель обзора: рассмотреть публикации по эпидемиологическим аспектам, клиническим особенностям заболеваний и тактике ведения поздних недоношенных детей.

Основные положения. По данным мировой литературы, количество поздних недоношенных детей составляет 70-80\% от числа всех преждевременно рожденных младенцев и не имеет существенной тенденции к снижению. Поздние недоношенные дети имеют сниженные компенсаторные возможности и более высокий риск заболеваемости и смертности по сравнению с таковым у доношенных детей. У поздних недоношенных чаще, чем у доношенных, регистрируются респираторные нарушения, нарушения терморегуляции, гипогликемия, гипербилирубинемия, инфекционные заболевания, нарушения во вскармливании.

Заключение. Знания о клинических особенностях адаптации и развития заболеваний позволяют неонатологам и педиатрам планировать тактику ведения и лечения поздних недоношенных детей.

Ключевые слова: недоношенные дети, поздние недоношенные, гестационный возраст, преждевременные роды, поздние преждевременные роды.
\end{abstract}

Конфликт интересов: автор заявляет об отсутствии возможных конфликтов интересов.

Для цитирования: Киосов А.Ф. Поздние недоношенные дети: эпидемиологические аспекты, заболеваемость, тактика ведения. Доктор.Ру. 2019; 9(164): 19-24. DOI: 10.31550/1727-2378-2019-164-9-19-24

\title{
Late Preterm Infants: Epidemiological Aspects, Morbidity, and Approaches to Medical Management
}

\author{
A.F. Kiosov \\ Regional Clinical Hospital No. 2; 18 Gagarin St., Chelyabinsk, Russian Federation 454010
}

\begin{abstract}
Objective of the Review: To review publications about the epidemiology of late preterm birth, specific clinical signs of diseases in late preterm infants, and approaches to the medical management of this group of premature newborns.

Key Points: According to the international literature, late preterm infants account for $70-80 \%$ of all preterm births, and this prevalence exhibits no significant tendency toward reduction. Late preterm infants have limited compensatory responses and are at higher risk of morbidity and mortality than full-term infants. Late preterm infants have a higher prevalence of respiratory disorders, temperature regulation problems, hypoglycemia, hyperbilirubinemia, infectious diseases, and feeding problems than full-term babies do.

Conclusion: An understanding of the specific clinical features of the adaptation process and the development of diseases in late preterm infants helps neonatologists and pediatricians plan approaches to the medical management and treatment of this population of babies.

Keywords: preterm infants, late preterm infants, gestational age, preterm delivery, late preterm delivery.
\end{abstract}

Conflict of interes: The author declares that he does not have any conflict of interests.

For citation: Kiosov A.F. Late Preterm Infants: Epidemiological Aspects, Morbidity, and Approaches to Medical Management. Doctor.Ru. 2019; 9(164): 19-24. (in Russian) DOI: 10.31550/1727-2378-2019-164-9-19-24

настоящее время к категории поздних недоношенных относят детей, появившихся на свет в результате преждевременных родов, произошедших на сроке от 34 0/7 до 36 6/7 недель или на сроке от 239 до 259 дней от первого дня последней менструации [1-4]. В зарубежной литературе поздних недоношенных детей в течение нескольких десятилетий определяли сразу несколькими терминами: late preterm, marginally preterm, moderately preterm, minimally preterm, mildly preterm $[1,4,5]$.

Наиболее широко долгое время использовался термин «почти доношенный» (near term) $[1,2,4,6]$. Однако употребление словосочетания «почти доношенный» часто приводило к недооценке рисков нарушения адаптации, заболеваемости и смертности у незрелого недоношенного ребенка $[1,2,7-9]$.
В 2006 г. Национальный институт здоровья детей и развития человека (National Institutes of Child Health and Human Development) в рамках семинара «0птимизация ухода и результатов для поздних недоношенных детей» (Optimizing care and outcome for late preterm infants) рекомендовал применять для детей, рожденных на сроке от 34 0/7 до 36 6/7 недель, термин «поздний недоношенный» (late preterm) $[1,2,4,5]$. Словосочетание «почти доношенный» (near term) теперь не рекомендуется использовать для детей данной группы. Понятие «поздний недоношенный» подчеркивает морфофункциональную незрелость таких детей по сравнению с доношенными [1, 3-6].

Доля родов, в результате которых появляются поздние недоношенные младенцы, составляет $4-15 \%$ от всех родов и $70-80 \%$ от всех преждевременных родов $[1,2,10-12]$.

Киосов Андрей Федорович - к. м. н., заведующий отделением патологии новорожденных и недоношенных детей ГБУЗ ОКБ № 2. 454010 , Россия, г. Челябинск, ул. Гагарина, д. 18. eLIBRARY.RU SPIN: 4863-1138. E-mail: kiosow@mail.ru 
Наиболее высокие показатели рождения таких детей отмечаются в странах азиатского региона и США [1, 3-5]. Частота рождения поздних недоношенных детей в Тайване - 10\%, в Турции - 13-15\% [12-14], в 2018 г. в США - 8\% [4]. Реже всего поздние недоношенные появляются на свет в европейских странах: в Швейцарии в 2017 г. их было 4,9\%, в Норвегии в 2016 г. - 4,3\% [15, 16].

За последние два десятилетия в мире увеличилось число поздних недоношенных новорожденных [1-5]. В азиатском исследовании в период с 2002 по 2008 г. отмечен рост количества таких детей с 8,6\% до 10\% [8], в США с 2014 по 2016 г. оно выросло с 6,82\% до 7,09\% [4]. Общемировая тенденция такова: увеличение числа поздних недоношенных в основном происходит за счет повышения рождаемости детей на сроке гестации 36 недель [17].

Рост распространенности поздних преждевременных родов в мире связывают с несколькими причинами: с широким использованием ВРТ, увеличением частоты медицинских вмешательств и оперативного родоразрешения, многоплодных беременностей; количества женщин с неблагоприятным акушерско-гинекологическим анамнезом и осложнениями течения беременности, а также с совершенствованием акушерской помощи и улучшением наблюдения за беременными женщинами $[1-6,10]$.

По мнению многих исследователей, наибольший вклад в рост числа поздних недоношенных детей вносит повышение распространенности кесарева сечения $[1,2,4,10]$. В США за последние 15 лет доля поздних недоношенных младенцев, рожденных путем кесарева сечения, выросла с 23,5\% до 34,3\% [4]. Частота кесарева сечения при поздних преждевременных родах в Канаде в 2014 г. составила $56,8 \%$ [9].

Значительную роль в увеличении количества поздних преждевременных родов играют отягощенный акушерский анамнез и осложнения течения беременности. A. Haroon и соавт. отмечают, что при поздних преждевременных родах у $26,1 \%$ матерей регистрируется высокая частота осложнений беременности [11]. По данным ФГБУ «НМИЦ АГП им. В.И. Кулакова» Минздрава России, к наиболее распространенным факторам, предрасполагающим к рождению поздних недоношенных детей, относятся угроза прерывания беременности на ранних сроках, урогенитальные инфекции, преэклампсия, многоплодная беременность, преждевременное излитие околоплодных вод [18].

у женщин при поздних преждевременных родах чаще, чем при родах в срок, наблюдаются гипертония (12,5\% против 1,5\%), СД (12,5\% против 9,2\%), инфекции мочевыводящих путей (1,5\% против 0,3\%) и длительный безводный период (8,9\% против 4,0\%) [11]. Согласно результатам швейцарского исследования, наиболее частым осложнением при самостоятельных поздних преждевременных родах была тяжелая преэклампсия (51,8\%), при оперативных поздних преждевременных родах - кровотечение (36,2\%) [15]. На сроке 34 недели регистрируется больше осложнений, чем на сроках 35-36 недель [19].

В австрийском исследовании с помощью регрессионного анализа определены значимые связи между материнскими факторами и перинатальными исходами при поздних преждевременных родах. Преэклампсия, преждевременное излитие околоплодных вод, отсутствие антенатального введения стероидов, кровотечение в родах, многоплодная беременность и мужской пол ребенка наиболее часто были связаны с высокими неонатальной заболеваемостью, часто- той госпитализации в реанимацию и длительностью лечения в стационаре [20].

Экономические затраты на выхаживание поздних недоношенных описаны в литературе недостаточно детально $[2,4,5]$. Поздние недоношенные требуют, конечно, меньше финансовых ресурсов, чем глубоко недоношенные. Тем не менее расходы на их выхаживание велики $[5,17,21]$. И связано это с тем, что поздних недоношенных значительно больше, чем недоношенных на сроке менее 34 недель $[1,5$, $10-12,17,21]$.

По данным канадского исследования 2014 г., наибольшие экономические затраты связаны с умеренно недоношенными и поздними недоношенными новорожденными [17]. Затраты в первые 10 лет жизни составили 67467 долларов США на одного раннего недоношенного, 52796 долларов на одного умеренно недоношенного и 10010 долларов на одного позднего недоношенного ребенка. Таким образом, общие затраты на ранних недоношенных детей - 123,3 млн долларов, на умеренно недоношенных - 255,6 млн долларов и на поздних недоношенных $-208,2$ млн долларов [17].

\section{СМЕРТНОСТЬ И ЗАБОЛЕВАНИЯ \\ ПОЗДНИХ НЕДОНОШЕННЫХ ДЕТЕЙ}

Поздние недоношенные дети имеют более высокий риск развития патологических состояний и заболеваний, а также неблагоприятных исходов, чем доношенные [4, 7-9, 22]. В австрийском исследовании 2014 г. заболеваемость у недоношенных новорожденных на 36 неделе составила $24 \%$, на 35 неделе - 43\%, на 34 неделе - 55\% [20]. По сведениям J.E. Williams и Y. Pugh, в 2018 г. уровень заболеваемости у детей с гестационным возрастом (ГВ) 34 недели был 51\% [4].

Неонатальная смертность среди поздних недоношенных значительно выше, чем среди доношенных новорожденных $[2,9,13]$, по данным M.L. Tsai и соавт., 0,3\% против 0,08\% [12]. A.L. Jefferies и соавт. приводят показатель неонатальной смертности поздних недоношенных 1,2\% [9], I.H. Celik и соавт. - 2,1\% [23]. В США неонатальная смертность среди таких младенцев в 4,5 раза выше, чем среди доношенных [24]. Она остается выше и в более старшем возрасте $[1,2,4,5]$.

Поздние недоношенные дети чаще умирают в первый год $[1,4,5]$. Показатель младенческой смертности в США среди поздних недоношенных детей был выше в 3 раза по сравнению с таковым среди доношенных (соответственно 7,7 и 2,5 на 1000) [4]. В канадском исследовании двухлетняя выживаемость ранних недоношенных составила 56\%, умеренно недоношенных - 92,8\%, поздних недоношенных $-98,4 \%$ [17].

Поздние недоношенные дети из-за рождения раньше срока на 4-6 недель не проходят важный период внутриутробного роста и развития [2-4]. Морфофункциональная незрелость органов и систем часто приводит у них к нарушениям постнатальной адаптации и к развитию различных заболеваний [1-4]. Наиболее часто у поздних недоношенных встречаются следующие патологические состояния и заболевания: дыхательные расстройства (респираторный дистресс, транзиторное тахипноэ), легочная гипертензия, апноэ, нарушения терморегуляции, полицитемия, трудности вскармливания, дегидратация, гипогликемия, гипербилирубинемия, внутриутробные и постнатальные инфекции, поражения ЦНС, задержки роста и неврологического развития $[1-4,6]$. Поздние недоношенные дети имеют высокий риск заболеваний и нарушений постнатальной адаптации, 
что необходимо учитывать при планировании тактики их ведения [1, 4-6].

В публикации A. Haroon и соавт. 2014 г. описывается, что у поздних недоношенных младенцев статистически значимо чаще, чем у доношенных, встречаются респираторный дистресс (16,5\% против 0,3\%), гипербилирубинемия, требующая фототерапии (37,9\% против $11,0 \%)$, сепсис (4,9\% против $0,3 \%)$ и нарушения роста (24,8\% против 4,0\%) [11].

B работе M.L. Tsai и соавт. среди поздних недоношенных и доношенных детей распространенность респираторного дистресс-синдрома составила 2,6\% и 0,02\% соответственно, сепсиса - 0,7\% и 0,2\%, гипогликемии - 3\% и 0,4\%, нарушений терморегуляции - 0,4\% и 0,05\%, затруднений во вскармливании - $2 \%$ и 0,4\%, гипербилирубинемии $14 \%$ и $3 \%$ [12].

По результатам азиатского исследования 2015 г., у поздних недоношенных с ГВ 34 недели частота различных патологий выше, чем у детей с ГВ 35-36 недель, а у них, в свою очередь, выше, чем у доношенных младенцев (табл.) [8].

Среди поздних недоношенных детей отмечается высокая частота задержки внутриутробного развития. По данным ФГБУ «НМИЦ АГП им. В.И. Кулакова» Минздрава России, она составляет среди недоношенных детей с ГВ 34 недели 20\%, при ГВ 35 недель - 20,1\%, при ГВ 36 недель - 12,9\%. Общая частота задержки внутриутробного развития среди поздних недоношенных детей - 16\% [18]. Тенденция такова, что с уменьшением ГВ увеличивается количество неонатальных заболеваний [8]. Согласно J.E. Williams и Y. Pugh, уровень заболеваемости в группе поздних недоношенных детей удваивается на каждую неделю ГВ менее 36 недель [4].

Большинство авторов отмечают, что поздние недоношенные имеют высокий риск респираторных нарушений $[7,8$, 10]. У них статистически значимо чаще возникают респираторный дистресс-синдром, персистирующая легочная гипертензия, а также потребность в терапии сурфактантом и терапии под постоянным положительным давлением в дыхательных путях, ИВЛ [2, 3, 24].

Частота дыхательных расстройств у поздних недоношенных детей увеличивается с уменьшением ГВ [10, 19], с ростом распространенности оперативного родоразрешения $[2,10]$. Респираторные нарушения при поздних преждевременных родах чаще регистрируются у мальчиков [10]; у детей, родившихся от матерей с высоким уровнем заболеваемости, которым не вводили профилактически кортикостероиды $[2,10]$, от первородящих и от женщин с избыточной массой тела [10].
Частота респираторного дистресса у младенцев на сроке 34 недели составляет 9\%, на сроке 35 недель - 4\%, на сроке 36 недель - 3\% [24]. Дети, рожденные в 34 недели, более часто нуждаются в проведении ИВЛ, чем новорожденные с ГВ 35 и 36 недель [19].

Поздним недоношенным чаще, чем доношенным, необходима госпитализация в отделение реанимации и интенсивной терапии (ОРИТ) $[2,8,12,19]$. Неинвазивная респираторная поддержка потребовалась $26 \%$ поздних недоношенных детей, ИВЛ - 10,7\%, из них 1,9\% - на фоне эндотрахеального введения сурфактанта [18].

В оказании специализированной медицинской помощи в условиях ОРИТ нуждались, по данным ФГБУ «НМИЦ АГП им. В.И. Кулакова» Минздрава России [18], 28,7\% поздних недоношенных. В работе M.P. De Carolis и соавт. таких детей было 17,1\% [19], согласно I.H. Celik и соавт. - 30\% [23], по результатам исследований A.L. Jefferies и соавт. $42 \%[9,25]$.

Поздним недоношенным чаще, чем доношенным младенцам, требуются реанимация после рождения $(21,4 \%$ против $1,2 \%)$ и лечение в ОРИТ (18,8\% против 2,4\%) [11]. В США получены подобные же данные по поступлению в ОРИТ: $33 \%$ против 7\% $(p<0,05)$ [24].

У поздних недоношенных детей по сравнению с доношенными также выше частота использования дыхательной поддержки Intermittent Positive Pressure Ventilation (дети с ГВ 34 недели - 15\%, с ГВ 33-36 недель - 3,5\%, доношенные дети - 1\%), парентерального питания (дети с ГВ 34 недели - 53\%, с ГВ 33-36 недель - 17\%, доношенные - 3\%), продолжительность пребывания в ОРИТ (дети с ГВ 34 недели $-14+22$ дня, с ГВ 33-36 недель $-4+4,7$ дня, доношенные $-2,6+3$ дня) [8]. Частота и продолжительность госпитализации в ОРИТ, длительность респираторной поддержки уменьшаются с увеличением ГВ $[8,19,26]$.

Исследование, проведенное в Тайване, показало, что распространенность госпитализации и длительность пребывания в ОРИТ у поздних недоношенных выше по сравнению с таковыми у доношенных новорожденных: $36 \%$ против $2 \%$ и 17 против 10 дней соответственно [12]. По данным A.L. Jefferies и соавт., респираторная поддержка была необходима 41,5\% поздних недоношенных младенцев. Продолжительность ее составляла 1,1-3,1 дня, средняя продолжительность пребывания в отделении реанимации - 8,1-9,3 дня [9].

у большинства поздних недоношенных детей, поступивших в ОРИТ, продолжительность интенсивной терапии невелика, многим детям не понадобились вмешательства $[9,25]$.

Частота разАичных патологий у поздних недоношенных и Аоношенных Аетей, \% [8]

\begin{tabular}{|l|l|l|l|}
\hline \multicolumn{1}{|c|}{ Патологии } & $\begin{array}{c}\text { Поздние недоношенные } \\
\text { с гестационным } \\
\text { возрастом 34 недели }\end{array}$ & $\begin{array}{c}\text { Поздние недоношенные } \\
\text { с гестационным } \\
\text { возрастом } \\
\text { 35-36 недель }\end{array}$ & \multicolumn{1}{|c|}{ Доношенные } \\
\hline Гипогликемия & 26 & 16 & 1 \\
\hline Нарушения терморегуляции & 5 & 1,7 & 0,2 \\
\hline Трудности во вскармливании & 30 & 9 & 1,4 \\
\hline Респираторный дистресс-синдром & 4 & 1 & 0,1 \\
\hline Транзиторное тахипноэ & 23 & 8 & 3 \\
\hline $\begin{array}{l}\text { Неонатальная желтуха, потребовавшая } \\
\text { лечения методом фототерапии }\end{array}$ & 63 & 24 & 8 \\
\hline
\end{tabular}


Согласно сведениям I.H. Celik и соавт., средняя длительность госпитализации в ОРИТ составила 7,5-9,1 дня [23].

Даже если поздние недоношенные дети не нуждаются в госпитализации в ОРИТ, большинству из них все же требуется специализированная помощь в отделениях патологии новорожденных $[5,9,18]$. По данным ФГБУ «НМИЦ АГП им. В.И. Кулакова» Минздрава России, 51,2\% поздних недоношенных необходимо выхаживание в условиях отделения патологии новорожденных и недоношенных детей, из них более половины - после завершения интенсивной терапии в условиях ОРИТ. Всем младенцам, родившимся на сроке 34 недели беременности, потребовалась специализированная медицинская помощь в условиях стационаров. Среди детей с ГВ 35 недель частота госпитализации составила $72,7 \%$, с ГВ 36 недель - 34,3\% [18].

Поздние недоношенные имеют и более высокий показатель повторной госпитализации в течение как неонатального, так и постнеонатального периода [2-4]. Факторами риска повторных госпитализаций являются юный возраст матери, первородящая мать, осложнения течения беременности и родов, исключительно грудное вскармливание при выписке из стационара [2, 4]. Согласно M.L. Tsai и соавт., у поздних недоношенных детей частота повторных госпитализаций составляет 4,4\%, тогда как у доношенных - только $2,3 \%$ [12].

Сравнение показателей заболеваемости новорожденных после выписки выявило, что желтуха и инфекции стали причинами всех повторных госпитализаций 77,1\% поздних недоношенных и 60,3\% доношенных младенцев $[4,8]$. Причинами повторных госпитализаций поздних недоношенных детей чаще всего становятся желтуха (71\%), подозрение на сепсис (20\%) и нарушения питания (16\%) [5].

Самый высокий риск повторных госпитализаций имели поздние недоношенные, которые сразу после рождения не были госпитализированы в отделения интенсивной терапии $[5,9,12,18]$. Поздние недоношенные дети, которые сразу после рождения не были в отделениях реанимации, в последующем в 2-3 раза чаще госпитализировались в стационар в связи с гипербилирубинемией по сравнению с доношенными новорожденными [4, 9]. Поздние недоношенные дети с короткими сроками пребывания в неонатальных отделениях также имеют более высокий риск повторной госпитализации $[4,9,12]$. У недоношенных с ГВ от 33 до 36 недель и длительностью пребывания в стационаре менее 4 дней выше показатели повторной госпитализации по сравнению с таковыми у остальных новорожденных [18].

В настоящее время многие авторы отмечают важность катамнестического наблюдения за поздними недоношенными детьми $[2,8,22]$. Исследования показывают, что у позднего недоношенного ребенка в последующем выше риск развития неврологических нарушений, расстройств речи, поведенческих отклонений, школьной дезадаптации и психических проблем $[1,2,21]$. Популяционные исследования свидетельствуют о том, что на каждую неделю снижения ГВ менее 37 недель постепенно увеличивается частота неблагоприятных исходов у детей [21].

В обзоре E. Kajantie и соавт. проведен анализ 53 работ, опубликованных в PubMed (48 исследований в странах северной Европы). По результатам этого обзора, люди, которые родились поздними недоношенными, часто умирают в возрасте моложе 45 лет. Ни одно из исследований не показало рост риска инсульта, однако все они обнаружи- ли повышенный риск СД 2 типа. Большинство работ также выявили высокий риск развития астмы и низкий - аллергического ринита [27].

При оценке поведенческих и эмоциональных отклонений у поздних недоношенных следует учитывать гендерные различия. У девочек увеличен риск возникновения эмоциональных проблем в возрасте 36 месяцев, у мальчиков нет [16]. Рожденные поздними недоношенными во взрослом возрасте имеют более низкие когнитивные способности, уровень образования, профессиональный статус и доход, у них чаще встречаются некоторые психические расстройства [27].

\section{ВЫПИСКА ПОЗДНИХ НЕДОНОШЕННЫХ ДЕТЕЙ ИЗ СТАЦИОНАРА, РЕКОМЕНДАЦИИ}

Сроки выписки из стационара позднего недоношенного ребенка индивидуальны и определяются совершенством терморегуляции, отсутствием соматических и инфекционных заболеваний, а также факторов социального риска $[2-5,28]$. В США поздних недоношенных детей, как правило, никогда не выписывают ранее 48 часов после рождения.

Перед выпиской витальные показатели должны быть в пределах возрастных значений в течение минимум 12 часов $[4,5,25,28,29]:$ частота дыхания - менее 60 вдохов в минуту, ЧСС - от 100 до 160 ударов в минуту, аксиллярная температура - от 36,5 до $37,4{ }^{\circ} \mathrm{C}$, самостоятельный стул - по крайней мере однократно. У позднего недоношенного ребенка также должны быть скоординированы сосание, глотание и дыхание.

Оценку грудного вскармливания, включая наблюдение за положением, следует проводить как минимум 2 раза в день после рождения. Любой ребенок с потерей веса за сутки более чем на 2-3\% от массы тела при рождении или с максимальной потерей веса 7\% и более от массы тела при рождении в роддоме должен быть оценен перед выпиской на предмет обезвоживания [4, 5, 25, 28, 29].

Необходимо определить риск развития гипербилирубинемии, проверить слух $[4,5,25,28,29]$. При клинических осмотрах ребенка не должно быть выявлено никаких отклонений, которые требуют продолжения госпитализации. Нужно произвести все необходимые анализы в соответствии с государственными правилами и требованиями, вакцинацию, скрининги матери и ребенка.

Многие исследователи заключают, что высокая частота повторных госпитализаций после выписки у поздних недоношенных детей может быть связана с недостаточным наблюдением за ними. Посещения младенца на дому после выписки из стационара уменьшают риск повторных госпитализаций $[4,5,25,28,29]$. Медицинский визит на дому должен быть организован после выписки новорожденного из больницы в течение 24-48 часов. Он нуждается в дополнительных посещениях на дому до тех пор, пока не будет достигнута стабильная положительная динамика веса $[4,5,25,28,29]$.

При разработке руководств по наблюдению за поздними недоношенными, в том числе по вопросам родоразрешения, методам респираторной поддержки, проблемам вскармливания и планирования выписки, рекомендуется учитывать практический опыт, полученный при работе с глубоко недоношенными детьми $[4,5,28-33]$.

Необходимо, чтобы план наблюдения за поздним недоношенным ребенком был понятен родителям и другим членам семьи. Результаты обследования ребенка обсуждаются с семьей или опекунами. Все последующие планы 
наблюдения должны быть определены и намечены совместно с родителями. В семье следует оценить социальные факторы риска, такие как токсикомания, жестокое обращение с ребенком, психические заболевания родителей, одинокая мать без социальной поддержки, мать-подросток, бездомность, риск насилия в семье.

Родители должны получить информацию и быть компетентны в следующих вопросах: расположение детской больницы (где будут осуществлять медицинскую помощь и наблюдение за ребенком), особенности грудного или искусственного вскармливания, уход за пуповиной, гениталиями, кожей ребенка, гигиена рук, использование термометра. Родители должны знать о том, как правильно одеть младенца по погоде на улицу, как обеспечить безопасность жилья, сна ребенка и положение во время сна. Их нужно проинформировать о необходимости использования автокресла для ребенка, об ограждении его от табачного дыма.

Из-за того, что поздние недоношенные дети подвергаются более высокому риску различных заболеваний и смерти,

\section{ЛИТЕРATУPA / REFERENCES}

1. Тимофеева Л.А., Шарафутдинова Д.Р., Шакая М.Н., Лазарева В.В. Поздние недоношенные: основные факторы риска и исходы (обзор). 2016; 7(25): 79-83. [Timofeeva L.A., Sharafutdinova D.R., Shakaya M.N., Lazareva V.V. Pozdnie nedonoshennye: osnovnye factory riska $i$ iskhody (obzor). 2016; 7(25): 79-83. (in Russian)]

2. Софронова Л.Н., Федорова Л.А., Кянксеп А.Н., Шеварева Е.А., Ялфимова Е.А. Поздние недоношенные - группа высокого риска ранних и отдаленных осложнений. Педиатрия. 2018; 97(1): 131-40. [Sofronova L.N., Fedorova L.A., Kyanksep A.N., Shevareva E.A., Yalfimova E.A. Pozdnie nedonoshennye - gruppa vysokogo riska rannikh i otdalennykh oslozhnenii. Pediatriya. 2018; 97(1): 131-40. (in Russian)]

3. Софронова Л.Н. Недоношенные дети, рожденные на поздних сроках гестации. Вестн. соврем. клин. медицины. 2014; 7(6): 89-92. [Sofronova L.N. Nedonoshennye deti, rozhdennye na pozdnikh srokakh gestatsii. Vestn. sovrem. kiln. meditsiny. 2014; 7(6): 89-92. (in Russian)]

4. Williams J.E., Pugh Y. The late preterm: a population at risk. Crit. Care Nurs Clin. North Am. 2018; 30(4): 431-43. DOI: 10.1016/j. cnc.2018.07.001

5. Engle W.A., Tomashek K.M., Wallman C.; Committee on Fetus and Newborn. "Late-preterm" infants: a population at risk. Pediatrics. 2007; 120(6): 1390-401. DOI: 10.1542/peds.2007-2952

6. Raju T.N. Developmental physiology of late and moderate prematurity. Semin. Fetal Neonatal Med. 2012; 17(3): 126-31. DOI: $10.1016 / j$.siny.2012.01.010

7. Mahoney A.D., Jain L. Respiratory disorders in moderately preterm, late preterm, and early term infants. Clin. Perinatol. 2013; 40(4): 665-78. DOI: 10.1016/j.clp.2013.07.004

8. Visruthan N.K., Agarwal P., Sriram B., Rajadurai V.S. Neonatal outcome of the late preterm infant (34 to 36 weeks): the Singapore story. Ann. Acad. Med. Singapore. 2015; 44(7): 235-43.

9. Jefferies A.L., Lyons E.R., Shah P.S., Shah V. Impact of late preterm birth on neonatal intensive care resources in a tertiary perinatal center. Am. J. Perinatol. 2013; 30(7): 573-8. DOI: 10.1055/s0032-1329685

10. Correia C., Rocha G., Flor-de-Lima F., Guimarães H. Respiratory morbidity in late preterm infants. Minerva Pediatr. 2018; 70(4): 345-54. DOI: 10.23736/S0026-4946.16.04580-1

11. Haroon A., Ali S.R., Ahmed S., Maheen H. Short-term neonatal outcome in late preterm vs. term infants. J. Coll. Physicians Surg. Pak. 2014; 24(1): 34-8. DOI: 01.2014/JCPSP.3438

12. Tsai M.L., Lien R., Chiang M.C., Hsu J.F., Fu R.H., Chu S.M. et al. Prevalence and morbidity of late preterm infants: current status in a medical center of Northern Taiwan. Pediatr. Neonatol. 2012; 53(3): 171-7. DOI: 10.1016/j.pedneo.2012.04.003

13. Binarbașı P., Akın Y., Narter F., Telatar B., Polatoğlu E., Ağzıkuru T. Mortality and morbidity in late-preterm newborns. Turk. Arch. Ped. 2013; 48(1): 17-22. D0I: 10.4274/tpa.821 их родителям требуется ознакомление со специальными инструкциями $[4,5,8,25]$. С помощью руководств родители знакомятся с принципами наблюдения за детьми после выписки из стационара [4, 5, 28-33]. Особенно важно ознакомить родителей поздних недоношенных с признаками гипербилирубинемии, симптомами обезвоживания и способами оценки питания [4, 5, 28-33].

\section{ЗАКЛЮЧЕНИЕ}

Поздние недоношенные по сравнению с доношенными детьми имеют более высокий риск развития нарушения адаптации и развития заболеваний. Наиболее часто у поздних недоношенных детей регистрируются нарушения терморегуляции, гипогликемия, дыхательные расстройства, апноэ, желтуха, инфекции, нарушения питания и обезвоживание. Сведения о клинических особенностях адаптации и развития заболеваний позволяют неонатологам, педиатрам планировать тактику ведения и лечения поздних недоношенных детей.

14. Karatas A., Albayrak M., Keskin F., Biyik I., Okur M., Gunes C. et al. Early neonatal outcomes in patients with late preterm birth. J. Turk. Society Obstet. Gynecol. 2013; 10(3): 165-72.

15. Bouchet N., Gayet-Ageron A., Lumbreras Areta M., Pfister R.E., Martinez de Tejada B. Avoiding late preterm deliveries to reduce neonatal complications: an 11-year cohort study. BMC Pregnancy Childbirth. 2018; 18(1): 17. DOI: 10.1186/s12884-017-1650-8

16. Stene-Larsen K., Lang A.M., Landolt M.A., Latal B., Vollrath M.E. Emotional and behavioral problems in late preterm and early term births: outcomes at child age 36 months. BMC Pediatr. 2016; 16(1): 196. DOI: 10.1186/s12887-016-0746-Z

17. Johnston K.M., Gooch K., Korol E., Vo P., Eyawo O., Bradt P. et al. The economic burden of prematurity in Canada. BMC Pediatr. 2014; 14: 93. DOI: 10.1186/1471-2431-14-93

18. Тимофеева Л.А., Киртбая А.Р., Дегтярев Д.Н., Шарафутдинова Д.Р., Цой Т.А., Карапетян А.О. и др. Поздние недоношенные дети: насколько они нуждаются в специализированной медицинской помощи? Неонатология: новости, мнения, обучение. 2016; 4: 94-101. [Timofeeva L.A., Kirtbaya A.R., Degtyarev D.N., Sharafutdinova D.R., Tsoi T.A., Karapetyan A.O. $i$ dr. Pozdnie nedonoshennye deti: naskol'ko oni nuzhdayutsya $\checkmark$ spetsializirovannoi meditsinskoi pomoshchi? Neonatologiya: novosti, mneniya, obuchenie. 2016; 4: 94-101. (in Russian)]

19. De Carolis M.P., Pinna G., Cocca C., Rubortone S.A., Romagnoli C., Bersani I. et al. The transition from intra to extra-uterine life in late preterm infant: a single-center study. Ital. J. Pediatr. 2016; 42(1): 87. DOI: 10.1186/s13052-016-0293-0

20. Scheuchenegger A., Lechner E., Wiesinger-Eidenberger G., Weissensteiner M., Wagner 0., Schimetta W. et al. Short-term morbidities in moderate and late preterm infants. Klin. Padiatr. 2014; 226(4): 216-20. DOI: 10.1055/s-0033-1355394

21. Vohr B. Long-term outcomes of moderately preterm, late preterm, and early term infants. Clin. Perinatol. 2013; 40(4): 739-51. DOI: 10.1016/j.clp.2013.07.006

22. Natarajan G., Shankaran S. Short- and long-term outcomes of moderate and late preterm infants. Am. J. Perinatol. 2016; 33(3): 305-17. DOI: 10.1055/s-0035-1571150

23. Celik I.H., Demirel G., Canpolat F.E., Dilmen U. A common problem for neonatal intensive care units: late preterm infants, a prospective study with term controls in a large perinatal center. J. Matern. Fetal Neonatal Med. 2013; 26(5): 459-62. DOI: 10.3109/14767058.2012.735994

24. Mally P.V., Hendricks-Muñoz K.D., Bailey S. Incidence and etiology of late preterm admissions to the neonatal intensive care unit and its associated respiratory morbidities when compared to term infants. Am. J. Perinatol. 2013; 30(5): 425-31. DOI: 10.1055/s-00321326989

25. Jefferies A.L.; Canadian Paediatric Society, Fetus and Newborn Committee. Going home: facilitating discharge of the preterm infant. Paediatr. Child Health 2014; 19(1): 31-42. 
26. Bassil K.L., Shah P.S., Shah V., Ye X.Y., Lee S.K., Jefferies A.L. Impact of late preterm and early term infants on Canadian neonatal intensive care units. Am. J. Perinatol. 2014; 31(4): 269-78. DOI: 10.1055/s-0033-1347364

27. Kajantie E., Strang-Karlsson S., Evensen K.A.I., Haaramo P. Adult outcomes of being born late preterm or early term - what do we know? Semin. Fetal Neonatal Med. 2019; 24(1): 66-83. DOI: 10.1016/j.siny.2018.11.001

28. Trembath A.N., Iams J.D., Walsh M. Quality initiatives related to moderately preterm, late preterm, and early term births. Clin. Perinatol. 2013; 40(4): 777-89. DOI: 10.1016/j.clp.2013.07.011

29. Quinn J.M., Sparks, M., Gephart S.M. Discharge criteria for the late preterm infant: a review of the literature. Adv. Neonatal Care. 2017; 17(5): 362-71. DOI: 10.1097/ANC.0000000000000406

30. Володин Н.Н., ред. Неонатология: национальное руководство. Краткое издание. М.: ГЭОТАР-Медиа; 2013. 896 с. [Volodin N.N., red. Neonatologiya: natsional'noe rukovodstvo. Kratkoe izdanie. M.: GEOTAR-Media; 2013. 896 s. (in Russian)]

31. Байбарина Е.Н., Дегтярев Д.Н., Зубков В.В., Рюмина И.И., Тимофеева Л.А. Клинические рекомендации. Базовая медицинская помощь новорожденному в родильном зале и в после- родовом отделении. М.: Ассоциация неонатологов; 2015. 33 c. [Baibarina E.N., Degtyarev D.N., Zubkov V.V., Ryumina I.I., Timofeeva L.A. Klinicheskie rekomendatsii. Bazovaya meditsinskaya pomoshch' novorozhdennomu $v$ rodil'nom zale $i v$ poslerodovom otdelenii. M.: Assotsiatsiya neonatologov; 2015. 33 s. (in Russian)]

32. Грошева Е.В., Дегтярева А.В., Ионов О.В., Ленюшкина А.А., Нароган М.В., Рюмина И.И. Клинические рекомендации. Энтеральное вскармливание недоношенных детей. М.: Ассоциация неонатологов; 2015. 28 c. [Grosheva E.V., Degtyareva A.V., Ionov O.V., Lenyushkina A.A., Narogan M.V., Ryumina I.I. Klinicheskie rekomendatsii. Enteral'noe vskarmlivanie nedonoshennykh detei. M.: Assotsiatsiya neonatologov; 2015. 28 s. (in Russian)]

33. Володин Н.Н., Дегтярев Д.Н., Дегтярева А.В., Карпова А.Л. Мебелова И.И., Пруткин М.Е. и др. Тактика ведения доношенных и недоношенных новорожденных детей с непрямой гипербилирубинемией. Клинические рекомендации. М.: Ассоциация неонатологов; 2016. 47 c. [Volodin N.N., Degtyarev D.N., Degtyareva A.V., Karpova A.L., Mebelova I.I., Prutkin M.E. i dr. Taktika vedeniya donoshennykh i nedonoshennykh novorozhdennykh detei s nepryamoi giperbilirubinemiei. Klinicheskie rekomendatsii. M.: Assotsiatsiya neonatologov; 2016. 47 s. (in Russian)] D

\section{ЧИТАЙТЕ} Dokmop.Py

\author{
недоношенные дети
}

Статьи схожей тематики в выпусках «Доктор.Ру» Педиатрия и «Доктор.Ру» Педиатрия Гастроэнтерология:

- Павлинова Е. Б., Сахипова Г. А. Бронхолегочная дисплазия у недоношенных детей: актуальность проблемы // Доктор.Ру. 2017. № 4 (133). С. 34-38.

- Лебедева 0.В., Чикина Т.А. Прогнозирование течения респираторного дистресс-синдрома у глубоко недоношенных новорожденных // Доктор.Ру. 2014. № 3 (91). C. 7-11.

- Рюмина И.И., Нароган М.В, Грошева Е.В., Дегтярева А.В. Трудные вопросы энтерального вскармливания недоношенных детей // Доктор.Ру. 2014. № 3 (91). С. 12-17.

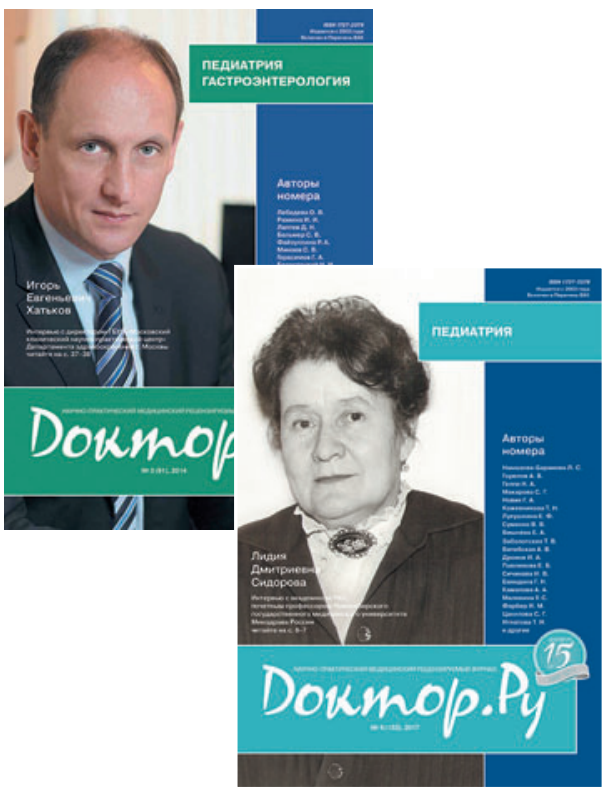

